\title{
Representações Sociais de Estudantes de Odontologia sobre o Atendimento
}

\section{em Clínica Odontopediátrica}

\author{
Elâine Cristina Vargas Dadalto* \\ Universidade Federal do Espírito Santo - UFES, Vitória, ES, Brasil \\ ORCID: https://orcid.org/0000-0002-1698-3326 \\ Edinete Maria Rosa** \\ Universidade Federal do Espírito Santo - UFES, Vitória, ES, Brasil \\ ORCID: https://orcid.org/0000-0003-4279-8308 \\ Zeidi Araújo Trindade*** \\ Universidade Federal do Espírito Santo - UFES, Vitória, ES, Brasil \\ ORCID: https://orcid.org/0000-0003-0549-5092
}

\begin{abstract}
RESUMO
O objetivo da pesquisa foi analisar as representações sociais construídas por estudantes de Odontologia sobre o atendimento prestado a crianças. A população-alvo do estudo foi constituída por estudantes de graduação em Odontologia da Universidade Federal do Espírito Santo (UFES), entrevistados no $10^{\circ}$ período, após terem frequentado as disciplinas de clínica odontopediátrica. As respostas às questões norteadoras foram registradas em áudio, submetidas à análise de conteúdo e interpretadas pela abordagem processual das

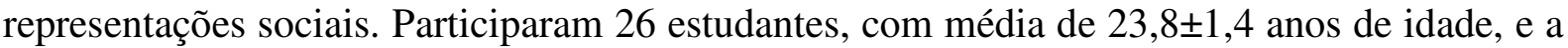
temática da entrevista semiestruturada envolveu expectativas prévias e vivência clínica. As representações que emergiram passaram pelo processo de organização em categorias. As representações sociais construídas por estudantes de Odontologia sobre o atendimento de crianças foram relacionadas à experiência gratificante e proveitosa, embora esta vivência possa ter sido complexa e desafiadora. Ademais, sob a ótica dos estudantes, é necessário ter paciência, ser compreensivo com a criança, manifestar carinho no atendimento, conquistar a confiança e usar a criatividade.
\end{abstract}

Palavras-chave: representação social, estudantes, odontologia, odontopediatria.

\section{Social Representations of Dental Students about Attendance in Pediatric}

\section{Dental Clinic}

\begin{abstract}
The objective was to analyze the social representations built by dental students about the care provided to children. The target population of the study was made up of undergraduate students in Dentistry from Universidade Federal do Espírito Santo (UFES), interviewed in the
\end{abstract}

ISSN 1808-4281 
10th period, after attending the disciplines of pediatric dentistry. The responses to guiding questions were recorded in audio, submitted to content analysis and interpreted by the procedural approach of social representations. Twenty-six students, with a mean of $23.8 \pm 1.4$ years of age, participated and the semi-structured interview theme involved previous expectations and clinical experience. The representations that emerged went through the process of organization into categories. The social representations constructed by dental students about the care of children were related to the rewarding and useful experience, although this experience may have been complex and challenging. In addition, from the perspective of the students, it is necessary to have patience, be understanding with the child, express affection in the attendance, gain confidence and use creativity.

Keywords: social representation, students, dentistry, pediatric dentistry.

\title{
Representaciones Sociales de Estudiantes de Odontología sobre la Atención
}

\section{en Clínica Odontopediátrica}

\begin{abstract}
RESUMEN
El objetivo fue analizar las representaciones sociales construidas por estudiantes de Odontología sobre la atención prestada a niños. La población objetivo del estudio fue constituida por estudiantes de graduación en Odontología de Universidade Federal do Espírito Santo (UFES), que se entrevistó en el $10^{\circ}$ período, después de cursar las disciplinas de odontopediatría. Las respuestas a preguntas orientativas se registraron en audio, sometidas al análisis de contenido e interpretadas por el enfoque procesal de las representaciones sociales. Participaron 26 estudiantes, con una media de 23,8 $2 \pm 1,4$ años, y la temática de la entrevista semiestructurada involucró expectativas previas y experiencia clínica. Las representaciones que surgieron pasaron por el proceso de organización en categorías. Las representaciones sociales construidas por estudiantes de Odontología sobre la atención de niños se han relacionado con la experiencia gratificante y provechosa, aunque esta vivencia pueda haber sido compleja y desafiante. Además, bajo la óptica de los estudiantes, es necesario tener paciencia, ser comprensivo con el niño, manifestar cariño en la atención, conquistar la confianza y usar la creatividad.
\end{abstract}

Palabras clave: representación social, estudiantes, odontología, odontología pediátrica.

O planejamento educacional para o graduando em Odontologia, reafirmado pela Resolução 3 do Conselho Nacional de Educação que institui as Diretrizes Curriculares Nacionais do curso de graduação em Odontologia, engloba o ensino de conteúdos teóricos e práticos de clínica odontopediátrica. Tais conteúdos se constituem em tema essencial para a formação do cirurgião-dentista, com perspectiva "generalista, humanista, crítica e reflexiva, para atuar em todos os níveis de atenção à saúde” (Resolução CNE/CES 3, 2002). 
O atendimento em clínica odontopediátrica no curso de graduação não está relacionado com habilidades inatas do futuro profissional para uma adequada relação com crianças. Para atender a esta demanda, o emprego de estratégias psicológicas para aumentar a colaboração da criança é tão importante quanto o preparo técnico do discente, que deve também cuidar da sua própria ansiedade frente à influência adversa de comportamentos não colaborativos (Possobon, Carrascoza, Moraes, \& Costa Jr., 2007).

Para o exercício do atendimento clínico a crianças, os graduandos em Odontologia podem apresentar alto nível de ansiedade devido à habilidade limitada para lidar com o comportamento de pacientes em estágios iniciais do desenvolvimento (Gerreth et al, 2019). O comportamento não colaborativo foi identificado como um dos principais fatores estressores no atendimento realizado por acadêmicos; os autores sugerem que o estresse vivenciado pelos discentes pode influenciar negativamente na qualidade e na execução do trabalho clínico (Vargas et al., 2013). Quando comparados a profissionais experientes, índices mais altos de estresse foram observados em acadêmicos (Davidovich, Pessov, Baniel, \& Ram, 2015).

A maioria das técnicas de manejo do comportamento infantil na clínica de odontopediatria apresentou boa aceitabilidade por graduandos do oitavo semestre, em comparação àqueles do início do curso de Odontologia; foi observado um aumento significante, no decorrer do curso, na aceitação das técnicas de controle de voz, modelação, uso de eufemismos, imobilização ativa, dentre outras, demonstrando que o aprendizado após cursar as disciplinas de atendimento odontopediátrico, em conjunto com a experiência clínica, influenciou na percepção dos discentes (Oliveira et al., 2015). Em outro estudo, as técnicas de reforço positivo e dessensibilização para orientação do comportamento infantil foram hierarquizadas pelos graduandos como mais aceitáveis, e as técnicas aversivas, incluindo controle de voz, foram menos aceitáveis (Al-Jobair \& Al-Mutairi, 2015).

Ao avaliar os pontos positivos da experiência na clínica odontopediátrica, graduandos de terceiro e quarto anos evidenciaram experiências agradáveis com as crianças, desde a descrição de comportamento cooperativo, até experiências pessoais de apego e uso de expressões carinhosas; como pontos negativos, os relatos versaram sobre abandono do tratamento de crianças não colaboradoras, falta de controle da mãe sobre seu filho e ausência às consultas agendadas (Vyawahare et al., 2013). O desenvolvimento de competências essenciais ao atendimento foi considerado determinante para aumentar o nível de confiança dos acadêmicos, o que foi atribuído à oportunidade de realizar procedimentos complexos em crianças nas atividades de extensão universitária (Rodd, Farman, Albadri, \& Mackie, 2010). 
Considerando a singularidade dos estudantes de Odontologia como um grupo social em processo de formação profissional, os estudos sobre a sua concepção e interpretação quanto ao atendimento odontológico prestado ao público infantil apresentam relevância para analisar se existe indicação para a instituição de atividades e medidas de redução do estresse, como estratégia do processo educacional para os acadêmicos que forem iniciar os primeiros atendimentos na clínica odontopediátrica. Para analisar e interpretar dados relacionados à concepção dos estudantes de Odontologia sobre o atendimento odontológico a crianças, o presente trabalho empregou a Teoria das Representações Sociais (TRS), proposta em 1961 (Moscovici, 1978), com análise e interpretação dos dados a partir da abordagem dimensional ou processual das representações (Jodelet, 2009).

A TRS engloba uma forma de conhecimento individual e partilhado por um grupo social, por meio da qual "[...] os homens tornam inteligível a realidade física e social, inserem-se num grupo ou numa ligação cotidiana de trocas [...]" (Moscovici, 1978, p. 28). As representações sociais se referem ao universo consensual e sua finalidade é tornar familiar algo desconhecido ou incomum, incluindo-o em uma categoria conhecida (Moscovici, 2017). A reconstrução de representações pode ser realizada por procedimentos de análise de conteúdo, inferindo a expressão dos contextos, com "tipos", "qualidades" e "distinções". As reflexões geradas podem traduzir valores, atitudes, estereótipos, símbolos e cosmovisões, que permitem traçar um perfil para identificar um contexto (Bauer \& Gaskell, 2000/2019).

Na concepção da abordagem dimensional ou processual, as representações sociais são fenômenos complexos que produzem, na constituição das subjetividades e da afirmação identitária, diversas dimensões que devem ser integradas em uma mesma apreensão. $\mathrm{O}$ indivíduo não deve ser concebido de maneira isolada, mas sim, como ator social ativo, afetado por diferentes aspectos da vida em um contexto social de interação. A forma de o indivíduo ver, pensar, conhecer, sentir e interpretar seu mundo, e sua existência nele, desempenha um papel indiscutível na orientação e reorientação das práticas sociais do grupo. O estudo das representações permite acessar os significados atribuídos a um objeto localizado no meio social e material, e examinar como os significados são articulados à sensibilidade, interesses, desejos, emoções e ao funcionamento cognitivo (Jodelet, 2009, 2018).

O presente estudo se dispôs a estudar as representações sociais de estudantes de Odontologia, de forma a subsidiar a estruturação de ações direcionadas a favorecer o enfrentamento do estresse ao iniciar o atendimento de crianças. Sendo assim, o objetivo foi analisar as representações sociais construídas por estudantes de Odontologia sobre o 
atendimento prestado ao público infantil, a partir das expectativas e da vivência do estudante após cursar as disciplinas de atendimento clínico cirúrgico-restaurador em crianças.

\section{Método}

A abordagem metodológica do presente estudo relaciona-se à pesquisa de campo descritiva, com amostragem não probabilística e de conveniência, no âmbito da pesquisa qualitativa. A investigação das representações sociais e de seus significados fundamentou-se na abordagem dimensional ou processual (Jodelet, 2009), de forma a analisar as expectativas e vivências dos estudantes no contexto da prática social.

\section{Participantes}

A população-alvo deste estudo foi constituída por estudantes do curso de graduação em Odontologia da Universidade Federal do Espírito Santo (UFES). Como critério de inclusão, o estudante deveria estar no $10^{\circ}$ período do curso, que corresponde ao semestre final de um total de cinco anos letivos, quando todas as clínicas de atendimento odontológico a crianças estivessem finalizadas, e estar disponível para participar da pesquisa. No currículo do curso de Odontologia da UFES, os estudantes têm o primeiro contato com o paciente infantil na disciplina de Saúde Bucal e Coletiva para atendimento preventivo. O primeiro tratamento cirúrgico-restaurador na faixa etária de 04-12 anos ocorre na disciplina de Odontopediatria, desenvolvida no $8^{\circ}$ período; na sequência, eles atendem crianças na disciplina do $9^{\circ}$ período, denominada Interdisciplinaridade em paciente infantil, enquanto a faixa etária de 0-3 anos recebe tratamento na disciplina optativa Clínica de Bebês.

\section{Instrumentos}

A coleta dos dados foi realizada por meio de entrevistas individuais, seguindo um roteiro semiestruturado contendo questões sobre idade do participante, sexo e convivência ou não com crianças. Três conjuntos de questões norteadoras auxiliaram o relato dos estudantes e versaram sobre sua vivência/experiência em relação à prestação do atendimento odontológico a crianças; suas expectativas; e possíveis problemas enfrentados. 


\section{Procedimento}

Coleta dos dados. Um teste-piloto foi executado previamente para verificar a compreensão das questões de acordo com o objetivo proposto. Foram entrevistados dois estudantes, um do sexo masculino e uma do sexo feminino, de forma a detectar se haveria alguma dificuldade na elaboração das respostas. Os resultados do teste-piloto demonstraram que o instrumento estava compatível e os termos empregados foram assimilados prontamente.

Os estudantes foram contatados no $10^{\circ}$ período, quando o projeto de pesquisa foi exposto, os objetivos foram esclarecidos, reforçando o caráter voluntário da participação e a garantia do sigilo do conteúdo da entrevista. $\mathrm{O}$ agendamento das entrevistas individuais ocorreu em horário previamente acordado, tendo sido esclarecido que não haveria respostas certas ou erradas, que o estudo era na área de Psicologia e que era importante a sinceridade nas respostas para retratar sua percepção sobre a vivência durante o atendimento clínico a crianças. A duração foi de cerca de 15 minutos e as respostas foram registradas em áudio.

Análise dos dados. Os dados referentes à caracterização dos participantes foram analisados por estatística descritiva. As respostas às questões norteadoras registradas em áudio foram submetidas ao processo de transcrição, e interpretadas por intermédio da análise de conteúdo, tendo a construção de módulos para categorização como referencial de codificação, o que aumenta a coerência e a fidedignidade (Bauer \& Gaskell, 2000/2019).

A partir do registro dos conteúdos principais destacados das respostas dos participantes às questões da entrevista, conforme a divisão temática proposta no roteiro do instrumento, foram realizadas categorizações iniciais para sistematizar e agrupar esse material. Para a análise e interpretação desses dados foi utilizada a abordagem processual das representações sociais, que permite a integração da subjetividade na construção e interpretação de indivíduos ou de um grupo social sobre a sua experiência cotidiana em determinado espaço público, exercendo a função de leitura da realidade, interpretação dos acontecimentos e guia de ação, formando um sistema de significações (Jodelet, 2018).

As representações sociais que emergiram após essa avaliação passaram pelo processo de organização em categorias, que constituíram o suporte de inferência para a análise das respostas dos estudantes de Odontologia quanto ao atendimento odontológico a crianças. Para ressaltar a representatividade das categorias com maior influência, aquelas com maior número de respostas foram indicadas na primeira posição no texto de descrição de cada categoria. 


\section{Considerações Éticas}

Em conformidade com a Resolução 466/12 do Conselho Nacional de Saúde, o projeto foi aprovado pelo Comitê de Ética em Pesquisa da Universidade Federal do Espírito Santo Campus Goiabeiras, sob o número CAAE: 56157616.8.3001.5542. Os estudantes que aceitaram participar assinaram o "Termo de Consentimento Livre e Esclarecido", contendo informações sobre os procedimentos, garantia de anonimato e confidencialidade dos dados.

\section{Resultados}

Foram entrevistados 26 estudantes do último período do curso de Odontologia, após terem prestado atendimento nas disciplinas de clínica odontopediátrica. A idade variou de 21

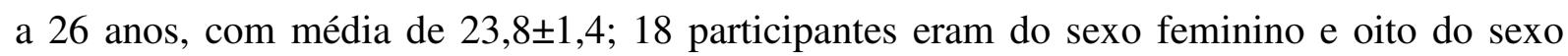
masculino. Considerando a convivência com crianças, após os participantes terem completado 16 anos de idade, 18 convivem ou já conviveram e oito nunca conviveram.

Após análise de conteúdo, as respostas às questões norteadoras foram submetidas à verificação para identificar as representações sociais. Os dados coletados permitiram a apreensão de conteúdo em seis categorias, descritas na sequência a seguir, que envolveram as expectativas prévias e a vivência/ experiência sobre o atendimento odontológico a crianças.

\section{O atendimento será difícil e estressante, mas dependendo da criança, poderá ser fácil, calmo e simples}

No imaginário dos estudantes, o atendimento odontológico a crianças seria difícil e estressante, não iriam conseguir atender porque as crianças seriam resistentes ao tratamento, ficariam com medo, iriam chorar o tempo todo, fazer birra e os pais precisariam segurá-las. Alguns relatos, em contrapartida, se referiram à expectativa prévia de que o atendimento seria fácil, calmo e simples, que o controle da criança seria uma tarefa fácil, que ela iria sentar e abrir a boca sob o comando do estudante como ocorre com o adulto. Essa narrativa foi encontrada com base nas respostas a respeito da expectativa antes da realização dos primeiros atendimentos clínicos em crianças. Por isso, observa-se que essas representações sociais estão ancoradas nas características das crianças e não na habilidade do profissional que as atende.

A maioria dos estudantes que tinha como expectativa que o atendimento seria difícil e estressante, manifestaram que a vivência durante a clínica odontopediátrica foi surpreendente 
por ter sido melhor do que o esperado e que aprenderam a lidar com as crianças aplicando conhecimentos adquiridos durante o desenvolvimento teórico-prático da disciplina de Odontopediatria; apenas um dos participantes que tinha a expectativa de que seria difícil, confirmou que a experiência foi exatamente dessa forma. Para os estudantes que imaginavam que o tratamento seria fácil, calmo e simples a experiência foi considerada muito difícil, de modo que passaram a valorizar mais o atendimento à criança, como exemplificado nos recortes dos relatos: "Tem que ter muita sabedoria para atender uma criança" (E09) ou "tem que ter muito dom para ser odontopediatra" (E23).

\section{Atender crianças é uma experiência gratificante, proveitosa, complexa e desafiadora}

As respostas sobre a vivência/experiência dos estudantes com os primeiros atendimentos odontológicos em crianças demonstraram claramente a influência do atendimento de crianças colaboradoras ou de crianças muito resistentes. As representações sociais constituídas na vivência em clínica odontopediátrica estão descritas a seguir.

$\mathrm{O}$ atendimento odontológico de crianças foi externado como uma experiência gratificante. Especialmente para os casos em que as crianças não colaboraram no início, os relatos realçaram a recompensa que os estudantes tiveram ao visualizar que houve evolução do tratamento, a partir do momento em que elas começaram a colaborar, de forma que essas crianças “... saíam abraçando, gostando, deixando tratar.” (E08). A inocência da criança e essa generosidade na demonstração de afeto positivo foi identificada pelos estudantes como fator de motivação e como vivência recompensadora, até mesmo como um aprendizado para a vida. Um dos estudantes descreveu o atendimento como atividade profissional prazerosa e outro demonstrou grande satisfação ao relatar sobre o agradecimento de uma criança que recebeu tratamento restaurador de uma fratura dentária: “... na escola, por ter um dente quebrado, um dente diferente, sofria bullying e foi um alívio para ele, assim, o tratamento.” (E21). Em quatro relatos ficou evidenciado que, embora houvesse dificuldade no atendimento, os respondentes consideraram a experiência gratificante.

Houve também a constatação de comoção ao verificar que a realidade quanto à situação precária de saúde bucal do público atendido não acontecia somente com adultos, de forma que os estudantes ficaram mais sensibilizados diante da necessidade de tratamento na criança do que no adulto. A partir da conquista da confiança da criança, consideraram ser possível conduzir o tratamento e realizar os procedimentos visando à melhoria da saúde bucal. 
Para outros estudantes, o atendimento foi considerado uma experiência proveitosa devido à auto avaliação de bom desempenho, à oportunidade de exercitar a paciência e ao aprendizado para obter o comportamento desejado da criança. Neste grupo, a maioria das crianças atendidas foi descrita como colaboradoras, tendo sido evidenciada a motivação do estudante diante da criança dócil e obediente.

Complementando os relatos desta categoria, alguns participantes que conviviam com crianças em ambiente domiciliar informaram que tal experiência facilitou a interação, de forma que eles tiveram uma boa atuação no atendimento odontológico infantil porque já tinham a vivência de entrar no ambiente lúdico, brincar e conversar com crianças, ao mesmo tempo em que conseguiam educar a criança para que ela ficasse quieta na cadeira odontológica. Além da convivência, o fato de ter participado da educação de uma criança pode ter reduzido a dificuldade, como no exemplo: “...tratei os pacientes da mesma forma que eu tratei meu irmão, que eu eduquei meu irmão, que eu convivi com ele, que eu falava que tem que fazer isso, tem que fazer aquilo, dava recompensa, ou não dava recompensa." (E14).

Outro grupo, entretanto, expressou que o atendimento de crianças foi uma experiência complexa e desafiadora, ficando evidente a dificuldade que encontraram para conseguir controlar o comportamento da criança ao fazer o atendimento. Os estudantes narraram situações que envolveram tensão e ansiedade e consideraram o tratamento odontológico de crianças como muito desgastante, pelo fato de precisar realizar os procedimentos e, ao mesmo tempo, falar muito para que a criança possa ter confiança e passar a colaborar; avaliaram, ainda, que o tratamento é desafiador pelo fato de ter que trabalhar sob muito estresse e realizar os procedimentos apressadamente, devido ao pouco tempo de colaboração da criança; ademais, observaram que o ambiente de trabalho é muito barulhento devido ao choro. Os estudantes também explicitaram a dificuldade encontrada no relacionamento com os pais da criança, tanto por estes não estimularem a cooperação do filho, quanto por não seguirem as orientações prestadas. Um participante ficou "desesperado" com a resistência de uma criança, enquanto outro se sentiu despreparado diante do choro de teimosia. Em suma, foi considerado mais difícil do que um tratamento em adultos.

Neste grupo de representações sociais os estudantes começam a reconhecer o papel do acadêmico no sucesso do atendimento. Portanto, as representações começam a ter influência do conteúdo que receberam no curso e da experiência prévia com crianças, apontando para uma ancoragem bidirecional em que as características das crianças podem sofrer influência da técnica e da habilidade do estudante, integrando as justificativas para sucesso no atendimento. 


\section{Para atender crianças é preciso ter paciência, criatividade, ser atencioso e carinhoso}

Como característica pessoal favorável para conseguir atender crianças, destacou-se a necessidade de paciência na análise das representações sociais. A paciência e a tranquilidade que é preciso para conversar com a criança, entreter, contar história, trabalhar dentro do tempo dela e insistir no convencimento para que ela possa colaborar com o tratamento, ou a paciência que é necessária para suportar o choro e não se estressar, evitando transparecer a frustração por não estar conseguindo realizar o procedimento odontológico.

Outros participantes realçaram habilidades que costumam aplicar no ambiente domiciliar como o ato de brincar com a criança na hora de apresentar a clínica, transformando o procedimento em uma brincadeira, e a facilidade para conversar usando a criatividade e a ludicidade que costumam aplicar com crianças da família para mudar o foco de atenção e explicar sobre os procedimentos de uma maneira que a criança possa entender, superando o medo. As características pessoais relacionadas ao comportamento atencioso e carinhoso também foram relatadas como favorável ao atendimento de crianças. Apenas um dos participantes relatou não ter habilidades com crianças.

Neste grupo de representações os estudantes demonstraram tomar para si a responsabilidade do sucesso na clínica, ainda que ancorados mais em habilidades pessoais do que habilidades sociais adquiridas ao longo do curso.

\section{O atendimento não dá certo quando sobrevém a impaciência, inexperiência e insegurança para impor limites}

As respostas relacionadas à característica pessoal desfavorável ao atendimento de crianças foram coesas em poucos itens, destacando-se a impaciência ao ter que ficar insistindo muito para que a criança colabore com o tratamento ou quando a criança quer fechar a boca no momento em que é preciso que ela mantenha a boca aberta, o que acaba gerando estresse, irritação ou intolerância, ocasionando o adiamento do tratamento ou a desistência por parte do estudante. Alguns relatos destacaram a inexperiência na comunicação com crianças, caracterizada por não saber se expressar em uma linguagem que a criança entenda, não conseguir lidar com crianças a ponto de passar confiança, não entender o universo infantil, e tratá-las como adulto, enquanto outros se referiram à insegurança, o que faz com que o atendimento não seja rápido, acreditando que é necessário domínio maior e conhecimento prático para atender crianças. 
Os demais relatos foram fragmentados em dez respostas diferentes: falta de habilidade para impor limites; compaixão relacionada ao medo de estar machucando a criança; exteriorização da expressão de frustração, não conseguindo disfarçar este sentimento diante do comportamento da criança; inflexibilidade, pelo fato de não ser maleável para adequar o tratamento às necessidades da criança; aparência muito séria, o que pode assustar uma criança; dificuldade para se expressar ao conversar com os pais da criança; pressa para fazer e terminar o procedimento, sem seguir os passos para ambientar a criança; ansiedade para acabar logo o procedimento porque a criança às vezes está agoniada; timidez como característica desfavorável que exigia muito esforço para começar um diálogo com a criança; e o fato de ser muito emotivo, ficando abalado diante do choro da criança.

Nesta categoria observa-se nitidamente que as representações sociais se afastam daquelas iniciais e agora se ancoram nas características próprias dos estudantes para justificar o seu insucesso no atendimento odontológico das crianças, sejam elas oriundas da falta de experiências prévias ou da falta de habilidades que poderiam ter sido adquiridas com a disciplina, como a habilidade para impor limites.

\section{$O$ atendimento pode resultar em emoção positiva pelo bem que se faz à saúde bucal da criança, ou emoção negativa pela desistência diante ao choro da criança}

Os episódios que os estudantes descreveram como evento marcante durante a vivência com os primeiros atendimentos do público infantil caracterizaram-se pela confiança das crianças no decorrer do tratamento, de forma que passaram a colaborar em procedimentos mais prolongados, relatado como uma conquista para conseguir a evolução do tratamento, o carinho das crianças e a emoção do estudante diante da reação comovente de uma criança que sorriu e chorou emocionada ao visualizar que seus dentes anteriores estavam restaurados.

Outros participantes descreveram sobre a impotência para agir quando os pais da criança não se manifestavam para estabelecer limites, culminando com a dispensa do paciente sem realizar o tratamento ou a manutenção da precariedade da saúde bucal por não seguir as orientações para cuidados domiciliares. A expressão de tristeza diante do choro incontrolável de pacientes de baixa idade durante procedimentos extensos foi realçada como evento marcante por alguns estudantes que frequentaram a disciplina optativa Clínica de Bebês, como pode ser constatado no relato: “(...) eu saí bastante triste por não ter conseguido controlar. Acho que foi a única vez que eu chorei. Ele chorava o tempo todo durante o tratamento de canal" (E04). 
Nesta categoria a representação social toma forma de consequência, positiva ou negativa, pelo trabalho realizado. Ela se ancora mais no resultado do atendimento do que no seu processo.

\section{Atender crianças é uma questão de aprendizado gradual, conquistado com a ajuda dos professores, dos colegas e de suas próprias habilidades em conquistar a confiança das crianças}

A maioria dos respondentes relatou que não enfrentou problema durante o atendimento clínico às crianças, o que foi atribuído ao aprendizado gradual pelo fato de estudar a teoria e ir desenvolvendo na clínica e de iniciar pelas técnicas de prevenção, fazendo a adequação da criança ao ambiente, antes de aumentar a complexidade dos procedimentos, à presença dos professores para auxílio sempre que necessário, tirando dúvidas, e à segurança de saber executar o procedimento como decorrência do processo ensino/aprendizagem.

Outros participantes atribuíram a inexistência de problemas durante o atendimento à paciência que tiveram para mostrar os instrumentais e reduzir a ansiedade das crianças, à construção da relação de confiança, conversando, tendo habilidade para lidar, de forma a criar vínculo com a criança, e à sorte de ter tido pacientes tranquilos. Foram relatadas também a contribuição do colega da dupla de trabalho e a cooperação positiva da mãe.

Um número pequeno de participantes relatou algum problema durante o atendimento clínico infantil, incluindo as crianças que não colaboravam adequadamente, de modo que a falta de seguimento e progresso do tratamento desestimulou esses estudantes. Dois deles comentaram sobre a agressividade da criança e um estudante relatou sobre o comportamento inconstante da criança.

Nesta última classe de representações observou-se que os estudantes ancoram suas representações de atendimento odontopediátrico em três pilares: aprendizagem durante o curso, características pessoais do atendente, e ajuda recebida de professores e colegas no momento da prática. Reconhecem a prática como a experiência que lhes permite contar com a ajuda de terceiros (professores e colegas), ampliando a análise do sucesso do atendimento de crianças, que antes se pautava no conteúdo recebido no curso e em suas próprias experiências que lhes conferiam características que facilitavam o relacionamento com as crianças. 


\section{Discussão}

Este estudo possibilitou o acesso aos significados atribuídos ao atendimento de crianças pelos estudantes de graduação em Odontologia da UFES, por meio da análise das suas representações sociais, utilizando a metodologia da pesquisa qualitativa (Bauer \& Gaskell, 2000/2019). Os dados se relacionam à análise das representações sociais construídas pelos estudantes sobre o atendimento prestado ao público infantil, a partir das expectativas e da vivência na clínica odontopediátrica. Os elementos de maior destaque da representação social sobre o atendimento odontológico à criança foram gratificante, proveitoso, complexo e desafiador, evidenciando a importância da aprendizagem teórica e da experiência.

Analisadas em referência ao contexto da vivência na clínica, sob a concepção da abordagem dimensional das representações sociais (Jodelet, 2009), as subjetividades constituídas na interpretação dos estudantes como atores sociais ativos configuraram a originalidade do presente trabalho e evidenciaram as interações destes com as crianças, os pais, o colega da dupla de trabalho e os professores. As afirmativas dos estudantes sobre o atendimento odontológico de crianças foram elaboradas ou complementadas partindo-se do princípio da sua experiência própria na infância, assim como pela circulação de expectativas e vivências quanto ao atendimento que foram motivo de conversas familiares. Outro ponto de influência na formação e circulação das representações sociais foi a opinião de estudantes que já cursaram a disciplina de Odontopediatria. Os participantes relataram que os alunos veteranos se referiam ao atendimento como sendo horrível e enfadonho, porque as crianças ficavam chorando, ou como a polarização entre o bom e o ruim, dependendo se o paciente a ser atendido seria colaborador ou não. Essas percepções compartilhadas por estudantes veteranos contribuíram para a formação das primeiras representações sociais dos estudantes que participaram deste estudo, como pode ser observado nos conteúdos presentes na primeira categoria aqui apresentada. O que leva à indagação de como os alunos veteranos, que já passaram pela formação em Odontopediatria, mantiveram sua representação ancorada nas características pessoais das crianças, diferentemente dos alunos participantes deste estudo que tiveram suas representações transformadas pela vivência da prática odontopediátrica.

As representações sociais como fenômenos que emergem no campo das ideias atuam como operadores simbólicos e lógicos da vida social, tendo em consideração que os fatos sociais são objetos de conhecimento (Jodelet, 2018). Em face da complexidade das interrelações que permeiam as disciplinas, a noção de representação figura como mediação para 
proporcionar uma visão global sob a ótica do estudante de Odontologia em relação à sua vivência durante a prática clínica.

A maioria dos estudantes se reportou à expectativa de que o tratamento de crianças seria difícil e estressante. Essa suposta dificuldade levou ao imaginário de que as crianças deveriam ser contidas para possibilitar o tratamento, o que não se confirmou na clínica, após cursar a disciplina de Odontopediatria. Outros autores evidenciaram que a restrição física foi indicada por acadêmicos para os casos de resistência da criança, recomendando treinamento sobre estratégias de manejo do comportamento para evitar seu uso (Batista et al., 2011).

O aprendizado gradual, a adequação da criança ao ambiente da clínica, o aumento gradativo da complexidade dos procedimentos, iniciando pela prevenção, e a presença dos professores para auxílio foram evidenciados como importantes para reduzir as dificuldades do tratamento em crianças e aumentar a segurança na execução dos procedimentos. De fato, o atendimento em clínica odontopediátrica não está relacionado com habilidades inatas dos graduandos para a interação com o paciente infantil, contudo sua compreensão melhora após cursar as disciplinas de clínica infantil, devido ao ensino e à vivência das estratégias psicológicas para aumentar a colaboração da criança (Oliveira et al., 2015) e à supervisão de docentes experientes para auxiliar no manejo do comportamento (Davidovich et al., 2015; Gerreth et al., 2019). Indubitavelmente, a confiança do paciente odontopediátrico deve ser estimulada pelos estudantes e, neste ponto, a abordagem odontológica preventiva tem o foco na redução da exposição à dor e do medo em relação ao tratamento (Serra-Negra et al., 2012).

A experiência dos estudantes na execução das técnicas odontológicas se mostrou diversificada, com a descrição de tratamentos de maior complexidade, a partir do aumento da confiança em lidar com o paciente infantil. Evidentemente, essa exposição dos acadêmicos para executar todos os procedimentos é necessária para desenvolver habilidades clínicas e competências essenciais, com o intuito de aumentar a confiança para atender crianças como profissionais generalistas (Rodd et al., 2010; Spiritoso et al., 2015; Sonbol, Abu-Ghazaleh, \& Al-Bitar, 2017), considerando que estes não devem necessariamente conhecer os pormenores de cada especialidade, mas ter uma visão geral para oferecer resolubilidade na maioria dos casos (Lage, Almeida, Vasconcelos, Assaf, \& Robles, 2017). Em contrapartida, tem sido discutido que a formação do cirurgião-dentista pode estar inadequada na prática clínica devido à redução do número de crianças na triagem das faculdades e à menor quantidade das necessidades de tratamento (Vyawahare et al., 2013; Casamassimo \& Seale, 2015), fato este que não se constatou como contratempo no presente estudo, devido à grande procura por atendimento de urgência odontopediátrica nessa mesma clínica (Muller et al., 2017). 
As representações sociais relacionadas à vivência do estudante de Odontologia na clínica de atendimento infantil demonstraram que foi uma experiência gratificante e proveitosa, mesmo para aqueles que tiveram dificuldades com algumas crianças. As representações que emergiram foram relacionadas à vivência recompensadora pelo afeto das crianças e à percepção do aumento da confiança no decorrer do tratamento, ao exercício da paciência e à sensibilização diante da situação precária de saúde bucal. Experiências agradáveis como fator de motivação dos estudantes, também foram registradas em associação ao comportamento cooperativo, apego e expressões carinhosas (Vyawahare et al., 2013).

A visão altruística dos estudantes quanto à empatia para ajudar a recuperar a saúde bucal das crianças mais carentes em recursos socioeconômicos, observada neste estudo, também foi constatada por outros autores, como questão importante na preparação da futura força de trabalho para que sejam profissionais socialmente responsáveis (Folayan et al., 2014; Santos, Madathil, Zuanon, Bedos, \& Nicolau, 2017). Por conseguinte, a convivência dos estudantes com a realidade tem o potencial de desencadear os sentimentos de empatia e alteridade, que podem influenciar positivamente na relação profissional-paciente, contribuindo na aplicação dos conceitos de humanização no atendimento (Bark et al., 2018), a fim de atingir objetivos quanto à formação generalista e humanista do cirurgião-dentista (Resolução CNE/ CES 3, 2002).

Em contraponto aos elementos gratificante e proveitoso, para um grupo de participantes a experiência com o atendimento de crianças contribuiu para o destaque dos elementos complexo e desafiador. As situações narradas envolveram tensão, ansiedade, irritação e tristeza diante do choro de crianças resistentes ao tratamento, tendo como representação que o atendimento odontológico de crianças é desgastante, estressante e difícil. Os principais pontos de dificuldade foram relacionados à insistência para a criança colaborar ou manter a boca aberta, ao comportamento inconstante, à inexperiência na comunicação e à insegurança associada à falta de prática. Este achado está em consonância com os resultados de pesquisas de outros autores em que os discentes se sentiam angustiados ao lidar com uma criança chorando (Nascimento et al., 2016); os principais fatores estressores nas aulas clínicas foram relacionados ao manejo do comportamento não colaborador (Vargas et al., 2013; Gerreth et al, 2019); e índices mais altos de estresse foram observados em universitários quando comparados a profissionais experientes (Davidovich et al., 2015).

Considerando que as representações sociais construídas nas interações individuais e coletivas estão em constante troca e circulação na vida cotidiana, atuando diretamente na orientação e reorientação das práticas sociais para constituir uma realidade consensual 
(Moscovici, 2017; Jodelet, 2018; Santos \& Dias, 2015), os estudantes poderão também participar na circulação das representações para os futuros alunos de clínica odontopediátrica. Ademais, a dimensão emocional do aprendizado tem figurado como tema na literatura recente sobre ensino odontológico (Leadbetter \& Gao, 2018). O grande desafio na clínica tem sido a introdução de metodologias que possam aumentar a autoconfiança dos discentes e prevenir comportamentos não colaborativos das crianças, como ferramenta complementar ao ensino por meio de explicações verbais; nesse sentido, o uso de imagens em vídeo (Slaven et al., 2019) e métodos interativos estão sendo indicados para melhorar as habilidades comunicativas dos graduandos, visando uma relação mais efetiva com o paciente infantil (Villar et al., 2017). Igualmente, a simulação em ambiente virtual de técnicas de comunicação apresentou potencial de aprendizado significante (Papadopoulos, Pentzou, Louloudiadis, \& Tsiatsos, 2013) e a visualização de imagens registradas em vídeo foi efetiva para aumentar a confiança dos acadêmicos quanto às técnicas de manejo do comportamento infantil para a anestesia local (Kenny, Alkazme, \& Day, 2018).

As representações sociais exercem uma função expressiva, constituem o produto de uma construção e são forjadas na interação com os discursos em circulação no grupo social (Jodelet, 2018). Destarte, os resultados do presente estudo apresentam contribuições relevantes para a área de Psicologia, fornecendo elementos para a análise da vivência do contexto do atendimento de crianças pelos estudantes de Odontologia de forma a dar subsídios para a elaboração de intervenções psicológicas preventivas para o enfrentamento do estresse durante o curso de graduação. Deve ser pontuado que o estresse e a exaustão emocional podem levar ao desenvolvimento da Síndrome de Burnout e influenciar negativamente no desempenho das atividades acadêmicas do curso e na qualidade do tratamento ao paciente (Jiménez-Ortiz et al, 2019). Procedimentos direcionados para reduzir o estresse são indicados, visando à melhoria no desempenho do estudante (Lin et al, 2020).

O panorama refletido neste estudo permite concluir que, após cursar as disciplinas de atendimento cirúrgico-restaurador em clínica odontopediátrica, as representações sociais construídas por estudantes de Odontologia sobre o atendimento de crianças foram relacionadas à experiência gratificante e proveitosa, embora esta vivência possa ter sido complexa e desafiadora. Ademais, sob a ótica dos estudantes, é necessário ter paciência, ser compreensivo com a criança, manifestar carinho no atendimento, conquistar a confiança e usar a criatividade.

Este estudo apresenta algumas limitações como a inclusão de somente um curso de Odontologia de universidade pública federal, o número menor de participantes do sexo 
masculino e o número reduzido de estudantes que nunca tinham convivido com crianças, o que mantém os resultados restritos ao contexto descrito. Certamente, uma amostra com maior expressividade e mais representativa do universo de estudantes permitiria maior validade externa. Recomenda-se para novos estudos o aumento e a diversificação da amostra. Outra sugestão é confrontar a técnica de explicações verbais de ensino das habilidades de direcionamento do comportamento da criança em comparação com as ferramentas suplementares como imagens de vídeo ou simulação virtual, visando investir no aumento da autoconfiança do estudante para a redução do estresse nos primeiros atendimentos à criança.

\section{Referências}

Al-Jobair, A. M., \& Al-Mutairi, M. A. (2015). Saudi dental students' perceptions of pediatric behavior guidance techniques. BMC Medical Education, 15(120), 1-9. doi: $10.1186 / \mathrm{s} 12909-015-0382-6$

Bark, M. M., Posanski, M., Oliveira, K. V., Brancher, J. A., Kriger, L., \& Gabardo, M. C. L. (2018). Alteridade e empatia: Virtudes essenciais para a formação do cirurgiãodentista [Alterity and empathy: Essential virtues for the formation of the dentist]. Revista da ABENO, 18(2), 104-113. doi: 10.30979/rev.abeno.v18i2.579

Batista, C. G., Nascimento, C. L., Rolim, G. S., Rocha, R. A. S. S., Rodrigues, A. F., Ambrosano, G. M. B., \& Moraes, A. B. A. (2011). Student self-confidence in coping with uncooperative behaviours in paediatric dentistry. European Journal of Dental Education, 15(4), 199-204. doi: 10.1111/j.1600-0579.2010.00656.x

Bauer, M. W., \& Gaskell, G. (2019). Pesquisa qualitativa com texto, imagem e som: Um manual prático (P. A. Guaresch, Trad.). Petrópolis, RJ: Vozes. (Obra original publicada em 2000).

Brasil (2002, março 4). Resolução CNE/CES 3, de 19 de fevereiro de 2002. Institui as Diretrizes Curriculares Nacionais do curso de graduação em Odontologia. Diário Oficial da União, Seção $1, \quad$ p. $10 . \quad$ Recuperado de http://portal.mec.gov.br/cne/arquivos/pdf/CES032002.pdf

Casamassimo, P. S., \& Seale, N. S. (2015). Adequacy of patient pools to support predoctoral students' achievement of competence in Pediatric Dentistry in U.S. dental schools. Journal of Dental Education, 79(6), 644-652. Recuperado de https://pubmed.ncbi.nlm.nih.gov/26034028/ 
Davidovich, E., Pessov, Y., Baniel, A., \& Ram, D. (2015). Levels of stress among general practitioners, students and specialists in Pediatric Dentistry during dental treatment. The Journal of Clinical Pediatric Dentistry, 39(5), 419-422. doi: 10.17796/1053-462839.5.419

Folayan, M. O, Sofola, O. O., Khami, M. R., Esan, A. O., Popoola, B. O., Orenuga, O. O., Folaranmi, N., Ligali, T. O., \& Phillips, A. S. (2014). Study motives, career choices and interest in paediatric dentistry among final year dental students in Nigeria. $B M C$ Medical Education, 14(130), 1-10. doi: 10.1186/1472-6920-14-130

Gerreth, K., Chlapowska, J., Lewicka-Panczak, K., Sniatala, R., Ekkert, M., \& BorysewiczLewicka, M. (2019). Self-Evaluation of Anxiety in Dental Students. BioMed Research International, 2019, 1-7. doi: 10.1155/2019/6436750

Jiménez-Ortiz, J. L., Islas-Valle, R. M., Jiménez-Ortiz, J. D., Pérez-Lizárraga, E., HernándezGarcıa, M. E., \& González-Salazar, F. (2019). Emotional exhaustion, burnout, and perceived stress in dental students. Journal of International Medical Research, 47(9), 4251-4259. doi: 10.1177/0300060519859145

Jodelet, D. (2009). O movimento de retorno ao sujeito e a abordagem das representações sociais. Sociedade e Estado, 24(3), 679-712. doi: 10.1590/S0102-69922009000300004

Jodelet, D. (2018). Ciências sociais e representações: Estudo dos fenômenos representativos e processos sociais, do local ao global. Revista Sociedade e Estado, 33(2), 423-442. doi: $10.1590 / \mathrm{s} 0102-699220183302007$

Kenny, K. P., Alkazme, A. M., \& Day, P. F. (2018). The effect of viewing video clips of paediatric local anaesthetic administration on the confidence of undergraduate dental students. European Journal of Dental Education, 22(1), e57-e62. doi: 10.1111/eje.12257

Lage, R. H., Almeida, S. K. T. T., Vasconcelos, G. A. N., Assaf, A. V., \& Robles, F. R. P. (2017). Ensino e aprendizagem em Odontologia: Análise de sujeitos e práticas. Revista Brasileira de Educação Médica, 41(1), 22-29. doi: 10.1590/1981$52712015 \mathrm{v} 41 \mathrm{n} 1 \mathrm{RB} 20150155$

Leadbetter, D., \& Gao, J. (2018). Engaging oral health students in learning basic science through assessment that weaves in personal experience. Journal of Dental Education, 82(4), 388-398. doi: 10.21815/JDE.018.041

Lin, X-J., Zhang, C-Y., Yang, S., Hsu, M-L., Cheng, H., Chen, J., \& and Yu, H. (2020). Stress and its association with academic performance among dental undergraduate 
students in Fujian, China: a cross-sectional online questionnaire survey. BMC Medical Education, 20(181), 1-9. doi: 10.1186/s12909-020-02095-4

Moscovici, S. (1978). Representação social da Psicanálise. Rio de Janeiro: Zahar.

Moscovici, S. (2017). Representações sociais: Investigações em Psicologia Social. Petrópolis: Editora Vozes.

Muller, C. E., Junior, M. F. S., Dadalto, E. C. V., Gomes, A. P. M., Sarmento, L. C., \& Gomes, A. M. M. (2017). Prevalence of odontogenic pain and associated factors in children treated at a pediatric dental emergency service. Revista Odonto Ciência, 32(3), 115-120. doi: 10.15448/1980-6523.2017.3.26352

Nascimento, M. M., Mugayar, L., Tomar, S. L., Garvan, C. W., Catalanotto, F. A., \& BeharHorenstein, L. S. (2016). The impact of an infant oral health program on dental students' knowledge and attitudes. Journal of Dental Education, 80(11), 1328-1336. doi: 10.1002/j.0022-0337.2016.80.11.tb06218.x

Oliveira, R. V. D., Ângelo, A. C. B., Brito, D. B. A., Medeiros, R. C. G., Forte, F. D. S., \& Sousa, S. A. (2015). Student's perceptions about pediatric dental behavior guidance techniques throughout a five-year dental curriculum. Brazilian Research in Pediatric Dentistry and Integrated Clinic, 15(1), 143-152. doi: 10.4034/PBOCI.2015.151.16

Papadopoulos, L., Pentzou, A., Louloudiadis, K., \& Tsiatsos, T. (2013). Design and evaluation of a simulation for Pediatric Dentistry in virtual worlds. Journal of Medical Internet Research, 15(10), e240. doi: 10.2196/jmir.2651

Possobon, R. F., Carrascoza, K. C., Moraes, A. B. A., \& Costa Jr, A. L. (2007). O tratamento odontológico como gerador de ansiedade. Psicologia em Estudo, 12(3), 609-616. doi: $10.1590 /$ S1413-73722007000300018

Rodd, H. D., Farman, M., Albadri, S., \& Mackie, I. C. (2010). Undergraduate experience and self-assessed confidence in paediatric dentistry: Comparison of three UK dental schools. British Dental Journal, 208(5), 221-225. doi: 10.1038/sj.bdj.2010.207

Santos, B. F. dos, Madathil, S., Zuanon, A. C. C., Bedos, C., \& Nicolau, B. (2017). Brazilian dental students' attitudes about provision of care for patients living in poverty. Journal of Dental Education, 81(11), 1309-1316. doi: 10.21815/JDE.017.088

Santos, G. T., \& Dias, J. M. B. (2015). Teoria das representações sociais: Uma abordagem sociopsicológica. PRACS: Revista Eletrônica de Humanidades do Curso de Ciências Sociais da UNIFAP, 8(1), 173-187. doi: 10.18468/pracs

Serra-Negra, J., Paiva, S. M., Oliveira, M., Ferreira, E., Freire-Maia, F., \& Pordeus, I. (2012). Self-reported dental fear among dental students and their patients. International 
Journal of Environment Research in Public Health, 9(1), 44-54. doi: 10.3390/ijerph9010044

Slaven, C. M., Wells, M. H., DeSchepper, E. J., Dormois, L., Vinall, C. V., \& Douglas, K. (2019). Effectiveness of and Dental Student Satisfaction with Three Teaching Methods for Behavior Guidance Techniques in Pediatric Dentistry. Journal of Dental Education, 83(8), 966-972. doi: 10.21815/JDE.019.091

Sonbol, H. N., Abu-Ghazaleh, S. B., \& Al-Bitar, Z. B. (2017). Undergraduate experience and self-assessed confidence in Paediatric Dentistry at the University of Jordan Dental School. European Journal of Dental Education, 21(4), e126-e130. doi: 10.1111/eje.12233

Spiritoso, S., Gross, E., Bean, C. Y., Casamassimo, P. S., Levings, K., \& Lloyd, P. (2015). Campus-based, community-based, and philanthropic contributions to predoctoral pediatric dental clinical education: Two years of experiences at one dental college. Journal of Dental Education, 79(8), 934-939. Recuperado de https://pubmed.ncbi.nlm.nih.gov/26246532/

Vargas, A. N., Fontes, B. N. V., Tolentino, A. B. Rodrigues, L. C. M., Oliveira, F. S., \& Castro, A. M. (2013). Percepção do estudante de Odontologia sobre os fatores estressores relacionados ao atendimento infantil. Faculdade de Odontologia de Lins/Unimep, 23(1), 11-19. doi: 10.15600/2238-1236/fol.v23n1p11-19

Villar, B., Montanet, G., Moreta, T., Zunino, M. M., Rodríguez, V., \& Pérez, D. M. (2017). Cómo mejorar las habilidades comunicativas en alumnos de Odontología. Cientifica Dental, 14(3), 193-199.

Vyawahare, S., Banda, N. R., Choubey, S., Parvekar, P., Barodiya, A., \& Dutta, S. (2013). Evaluation of undergraduate clinical learning experiences in the subject of pediatric dentistry using critical incident technique. Journal of Indian Society of Pedodontics and Preventive Dentistry, 31(2), 100-106. doi: 10.4103/0970-4388.115710

\footnotetext{
Endereço para correspondência

Elâine Cristina Vargas Dadalto

Universidade Federal do Espírito Santo

Programa de Pós-Graduação em Psicologia da UFES

Avenida Fernando Ferrari, 514, Campus Goiabeiras, Vitória - ES, Brasil. CEP 29075-910

Endereço eletrônico: elainedadalto@gmail.com
} 


\title{
Edinete Maria Rosa
}

Universidade Federal do Espírito Santo

Programa de Pós-Graduação em Psicologia da UFES

Avenida Fernando Ferrari, 514, Campus Goiabeiras, Vitória - ES, Brasil. CEP 29075-910

Endereço eletrônico: edineter@gmail.com

\section{Zeidi Araújo Trindade}

Universidade Federal do Espírito Santo

Programa de Pós-Graduação em Psicologia da UFES

Avenida Fernando Ferrari, 514, Campus Goiabeiras, Vitória - ES, Brasil. CEP 29075-910

Endereço eletrônico: zeide.trindade@gmail.com

Recebido em: 29/03/2019

Reformulado em: 07/08/2020

Aceito em: $13 / 08 / 2020$

\author{
Notas \\ * Doutorado e Pós-Doutorado em Psicologia pela Universidade Federal do Espírito Santo (UFES), Mestrado em \\ Odontopediatria (UFRJ), Professora de Odontopediatria (UFES) \\ ** Doutorado em Psicologia (USP), Pós-doutora (UNCG), Professora do Departamento de Psicologia Social e \\ do Desenvolvimento e do Programa de Pós-Graduação em Psicologia (UFES). \\ *** Doutorado e Pós-Doutorado em Psicologia (USP), Professora Titular aposentada da Universidade Federal do \\ Espírito Santo e do Programa de Pós-Graduação em Psicologia (UFES).
}

Este artigo de revista Estudos e Pesquisas em Psicologia é licenciado sob uma Licença Creative Commons Atribuição-Não Comercial 3.0 Não Adaptada. 